\title{
The integral field spectrograph TIGER: results and prospects
}

\section{R. Bacon}

\section{Observatoire de Lyon, 69561 Saint-Genis-Laval Cedex, France}

\begin{abstract}
The integral field spectrograph TIGER, in operation at CFHT since a few years, has been successfully used on a large variety of astrophysical objects, ranging from the planet Mars to distant radio-galaxies and quasars. This review discussed the peculiarities of the TIGER design and gives the main characteristics of the instrument. Two examples of scientific applications are presented to highlight the new possibilities offered by integral field spectrography. Finally the OASIS project dedicated to the CFHT adaptive optics bonnette is briefly presented.
\end{abstract}

\section{Introduction}

In $1982, \mathrm{G}$. Courtès proposed an elegant solution to achieve spectrographic analysis of extended objects with a full 2D spatial coverage. This concept, based on a micro-lenses array (see Section 2), allows to retreive three dimensions (2D space and 1D wavelength) information on a $2 \mathrm{D}$ detector without the loss of sensitivity associated with time-scanning schemes. This multiplex property presents many advantages, which makes this concept particularily well suited for low to medium spectral resolution studies of faint object combined with a high spatial resolution capability.

The integral field spectrograph TIGER ${ }^{1}$ is the first realization of this concept. Since its first light at the CFH $3.6 \mathrm{~m}$ telescope in 1987, TIGER has undergone only a few substantial optical and electro-mecanical modifications. It operates regularily at CFHT since the end of 1990 , and has been used by six different teams on a large variety of objects ranging from planet Mars to distant radio-galaxies.

The successful first test of the TIGER design originated from the joint effort of G. Courtès, Y. Georgelin, G. Monnet and the author with the help of G. Lelièvre, executive director of CFHT at that time, who kindly offered some discretionary time. However the realisation of an instrument based on such an original concept is a complex and long lasting operation. Its success belongs also to all of those who joined the scientific team or participated to its technical

\footnotetext{
${ }^{1}$ The prototype was nicknamed TIGER because it has to share the same cage (focal reducer) than a PUMA (for PUnching MAchine, the ancestor of the MOS spectrograph). The french acronym is "Traitement Intégral des Galaxies par l'Etude de leurs Raies".
} 


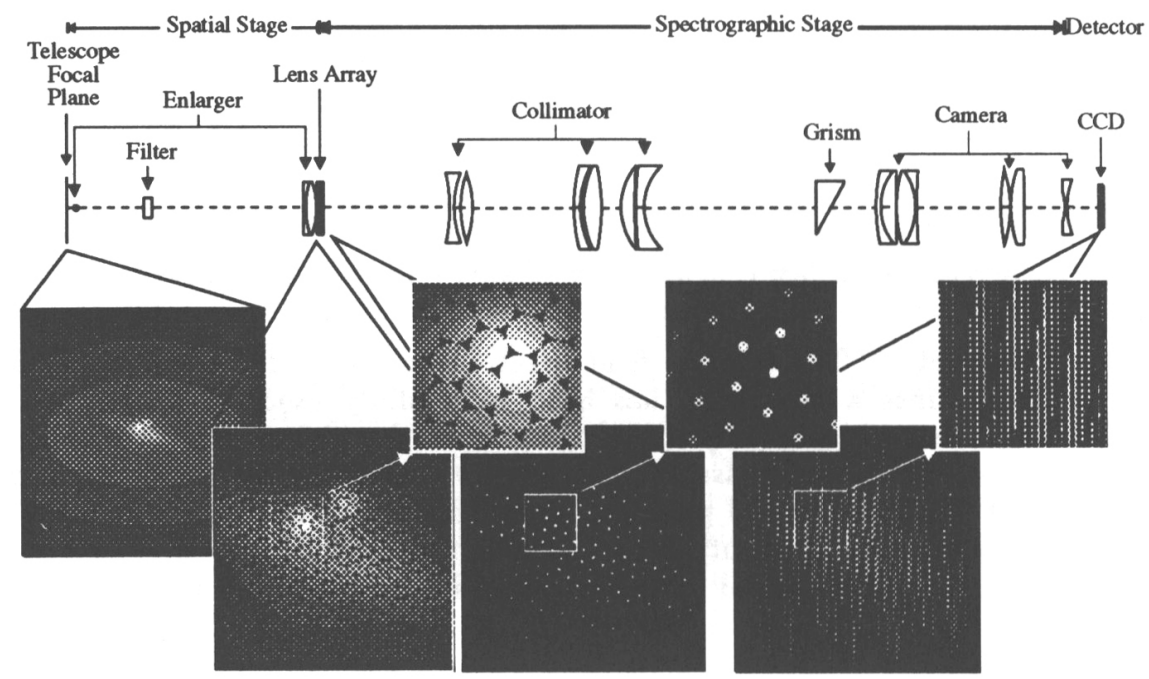

Figure 1. TIGER optical design

realisation: G. Adam, A. Baranne, D. Dubet, J.P. Dubois, E. Emsellem, P. Ferruit, E. Pécontal, A. Rousset, F. Sayede.

Some technical information about TIGER can be found in Courtès et al., 1987 and Bacon et al., 1988). A more complete discussion about the TIGER design as well as about its optical performances will be found in a forthcoming paper (Bacon et al., 1995).

\section{Optical design}

The schematic layout of the optical configuration is illustrated in Fig.1. The field of view (image $A$ in Fig.1) is enlarged and focused on the micro-lenses array by an objective and a field lens. The array performs the spatial sampling (image $B$ in Fig.1). Each micro-lens gives an image of the exit pupil (image C in Fig.1) whose size is much smaller $(\sim 45 \mu \mathrm{m})$ than the input lens diameter $(1.4 \mathrm{~mm})$. The remaining part is a classical grism-based spectrograph. Each micro-pupil, dispersed by the grism, gives a spectrum on the CCD (image D in Fig.1). A slight rotation between the dispersion axis and the micro-lenses array, combined with a wide band interference filter, avoids overlapping between spectra. Note that, contrarily to the ARGUS design, where a fibers bundle is used to re-arrange spectra on a pseudo-slit, only classical refractive optics is used on the TIGER design. This has a few advantages, as the conservation of the aperture ratio and the possibility to have a complete sampling (hexagonal or square lenses) without light-loss. However, as it will be seen later, the two designs are quite complementary.

The TIGER design deserves a few comments: 
- Contrarily to classical spectrographs, the pupil rather than the field is imaged on the detector. This allows the spectrographic point spread function to be independent of the spatial content of the field.

- Each spectrum is well separated ( 7 pixels) from its neighbours. This ensures that optical aberrations of the spectrograph will not degrade the spatial resolution.

- As for any classical multi-slit spectrographs, some spectra are truncated at the edge of the detector. It follows that the spectral length in pixel should be limited to a fraction of the detector linear size in order to avoid too large a fraction of truncated spectra.

One important parameter in this design is the separation between two adjacent spectra $(\delta)$. The ratio of this parameter to the cross-dispersion PSF should be large enough to avoid any cross-talk beween neighbouring spectra. However the larger $\delta$ is, the smaller the number of spectra $(N)$ will be. Given the number of pixel $(n \times n)$ of the detector, $N$ is given by $N=n^{2} / \delta l(l$ being the spectral length in pixels). The fraction of truncated spectra is then $k=l / n$.

Practically $\delta=7$ pixels is used (assuming a 2 pixels FWHM cross-dispersion PSF). Then taking $k<0.25$ gives $l>0.25 n$ and $N>0.57 n$. These values show that the TIGER design favors spatial rather than spectral coverage. It is quite complementary to the ARGUS design in that respect.

\section{Optical and observational characteristics}

TIGER's spatial characteristics have been chosen to match the current spatial resolution at CFHT, namely 0.7 arcsec FWHM $70 \%$ of the time. The spectral resolution is limited on the higher end by the maximum Grism path difference $(\sim 15 \mathrm{~mm})$ and at the lower end by the intrusion of strong zero order in the CCD area.

Its optical parameters have been optimized for the CFHT SAIC CCD chip of $1024^{2} \times 18 \mu \mathrm{m}$ pixels. The optical computation of the spectrograph (collimator + camera) has been performed by A. Baranne. Table 3. gives the general optical characteristics, while Table 3. gives the corresponding observational parameters.

\section{Data processing}

A major effort has been put in the development and realisation of new algorithms to handle efficiently the specificities of the TIGER data. The whole package has been regularily used at the Observatoire de Lyon since 1992. A new documented, user-friendly, X11-Motif package is now under development for the TIGER mode of the OASIS project (see Sect.6.).

The most specific task of this data reduction package is spectra extraction from the preprocessed CCD frame. Most of the remaining procedures are quite common in any $3 \mathrm{D}$ data processing. However, we had to develop most of them because of the lack of true 3D data reduction handling in the main images 


\begin{tabular}{|c|c|}
\hline Wavelength range & $0.35-1 \mu \mathrm{m}$ \\
\hline $\begin{array}{l}\text { Imagery enlarger focal length } \\
\text { Imagery enlarger aperture ratio }\end{array}$ & $\begin{array}{r}40 \mathrm{~mm} \\
f / 42\end{array}$ \\
\hline $\begin{array}{l}\text { Spectrographic enlarger } 1 \text { focal length } \\
\text { Spectrographic enlarger } 1 \text { aperture ratio }\end{array}$ & $\begin{array}{r}10 \mathrm{~mm} \\
\mathrm{f} / 206 \\
\end{array}$ \\
\hline $\begin{array}{l}\text { Spectrographic enlarger } 2 \text { focal length } \\
\text { Spectrographic enlarger } 2 \text { aperture ratio }\end{array}$ & $\begin{array}{r}15 \mathrm{~mm} \\
\mathrm{f} / 132 \\
\end{array}$ \\
\hline $\begin{array}{l}\text { Micro-lenses array } \\
\text { Micro-lenses diameter } \\
\text { Micro-lenses focal length } \\
\text { Micro-lenses aperture ratio }\end{array}$ & $\begin{array}{r}564(24 \times 21) \text { lenses }^{a} \\
1.39 \mathrm{~mm} \\
11.4 \mathrm{~mm} \\
\mathrm{f} / 8.2\end{array}$ \\
\hline $\begin{array}{l}\text { Collimator focal length } \\
\text { Collimator aperture ratio }\end{array}$ & $194 \mathrm{~mm}$ \\
\hline Grism & 150 to 600 groves \\
\hline $\begin{array}{l}\text { Camera focal length } \\
\text { Camera aperture ratio }\end{array}$ & $138 \mathrm{~mm}$ \\
\hline CCD & $1024^{2} \times 18 \mu \mathrm{m}$ \\
\hline Total transmission & 0.12 \\
\hline
\end{tabular}

Table 1. TIGER optical characteristics.

${ }^{a}$ Only 450 lenses are imaged on the detector

\begin{tabular}{|c|r|r|}
\hline Spatial sampling & Field of view & \\
\hline 0.39 arcsec. & $7 \times 7$ arcsec $^{2}$ & \\
0.61 arcsec. & $10 \times 10$ arcsec $^{2}$ & \\
\hline Spectral resolution & Central wavelength & Wavelength range \\
\hline 1300 & $5010 \AA$ & $540 \AA$ \\
1800 & $6850 \AA$ & $540 \AA$ \\
370 & $6320 \AA$ & $2400 \AA$ \\
540 & $7575 \AA$ & $2400 \AA$ \\
\hline
\end{tabular}

Table 2. TIGER observational characteristics. 
processing systems dedicated to optical astronomy (e.g. IRAF, MIDAS). All these programs were developed under the MIDAS architecture.

Here I only briefly describe the bases of the spectra extraction. Further details will be found in a forthcoming paper (Rousset et al., 1995).

As shown in Sect.2., multiple images of the telescope pupil are imaged on the detector and thus the spectrum PSF is independent of the object. We use this property to fit a model of the cross-dispersion PSF on a high signal to noise ratio flat-field exposure, which is then used to extract the intensity along each spectrum.

In practice, the cross-dispersion PSF is modelled as the convolution product of the micro-pupil (image of the telescope primary mirror with its central obscuration) integrated along the dispersion direction $(P(x))$ with a parametric approximation of the focal reducer PSF (generally a Gaussian or a Lorentzian function gives a good approximation). The result is then binned in CCD pixels $(\Delta)$. We finally get e.g. for a Gaussian function:

$$
P S F(x)=\int_{x-0.5 \Delta}^{x+0.5 \Delta} P(t) \otimes I_{0} \exp \left(-\frac{\left(t-x_{0}\right)^{2}}{2 \sigma_{0}^{2}}\right) d t
$$

To take into account possible overlap between neighbouring spectra we fit simultaneously each spectra with its two neighbours and save the two PSF parameters $\left(x_{0}, \sigma_{0}\right)$ of the central one only. The size of the micro-pupil varies with the focal length of the enlarger, and the focal reducer PSF changes with wavelength, but for a given instrumental configuration (i.e. the same enlarger and grism), the fitted PSF parameters are common to all exposures. They are used to compute the intensity as function of wavelength for each spectrum, using a: linear fit of the intensity of this spectrum and its two neighbours. This process is quite robust and minimize cross-talk bewteen adjacent spectra.

\section{Example of scientific targets}

Rather than giving en exhaustive list of the scientific targets that have been observed with TIGER for the last few years ${ }^{2}$, I will focus on some examples to highlight the new possibilities offered by integral field spectrography.

\subsection{The gravitationnal lens $2237+0305$ (Einstein cross)}

In this first example we show how to use the spectral information to disentangle the images of the quasar from the galaxy. These results are taken from the recent Fitte and Adam (1993) paper.

One exposure taken in September 1991, gave 400 spectra covering the central part of the galaxy with an 0.4 arcsec spatial sampling. One of these spectrum located near one of the quasar image is presented in Fig.2. Clearly identified is the redshifted CIII] emission line of the quasar.

Integrating the flux over the whole spectral range for each spectra allows to reconstruct the equivalent of a direct image through the same filter. This image

\footnotetext{
${ }^{2}$ Most of the major scientific results obtained so far with TIGER are presented in this conference.
} 


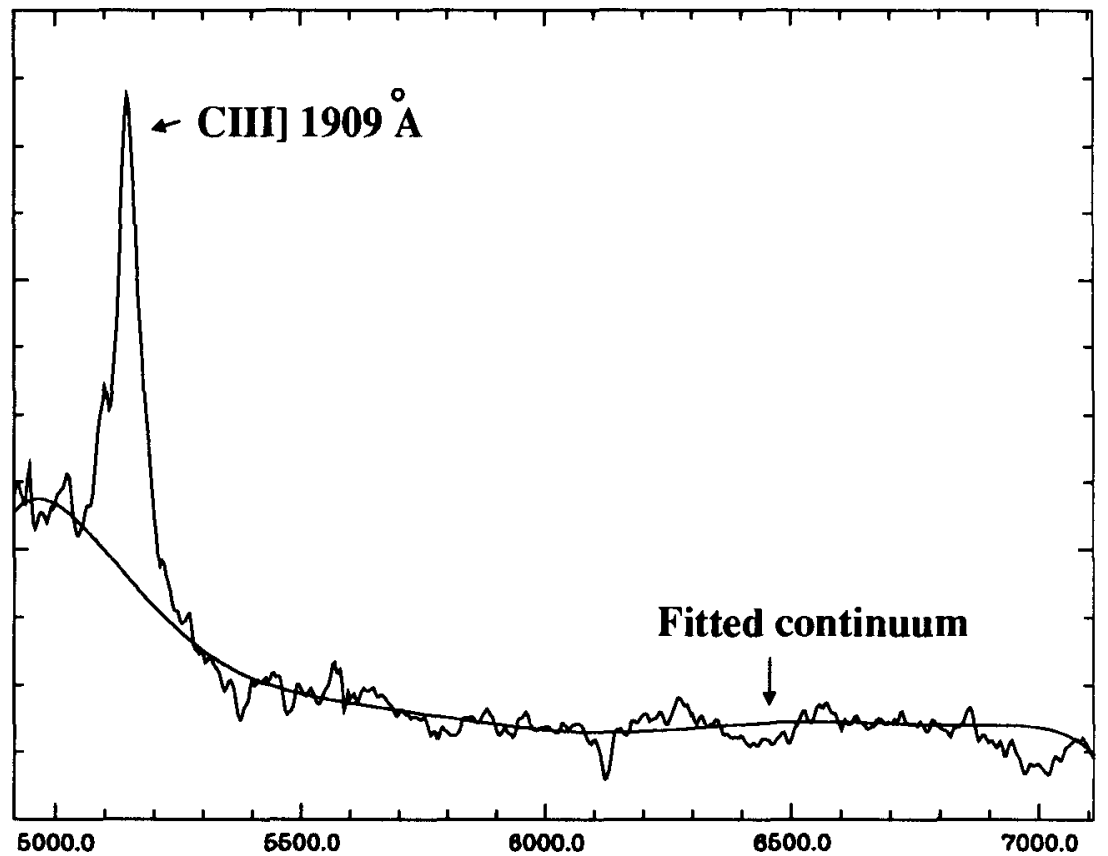

Figure 2. Einstein cross typical spectrum

(Fig.3) shows the well known cross-shaped of this object with five maxima, the central one being the nucleus of the lens galaxy.

This image is obviously dominated by the continuum which is an unknown combination of the quasar and galaxy fluxes. However it is quite easy to substract properly the continuum independently for each spectrum (see Fig.2). This continuum free CIII] reconstructed image, displayed in Fig.3 (right panel), allowed Fitte and Adam to switch off the galaxy, letting the sole quasar images. From such images they were able to measure micro-amplification lensing effects, as well as to study the galaxy morphology.

One can argue that direct narrow band imaging would essentially give the same result. However this would be possible only if the CIII] emission line is already detected (i.e. the spectra is already in hand). Furthermore a continuum estimation which uses at most two narrow bands adjacent to the emission line wavelength, cannot be as accurate as a fitting taking into account 200 resolved spectral elements. Note also that when spatial resolution is crucial $^{3}$, seeing variations between the continuum and the emission line exposures can affect strongly the results. In TIGER, the multiplex advantage ensures the same spatial resolution, sampling and absolute position for all quantities derived from the spectra.

\footnotetext{
${ }^{3}$ It was indeed the case in this observation, with a separation of only 0.7 arcsec. between the nuclei and the nearest quasar image
} 

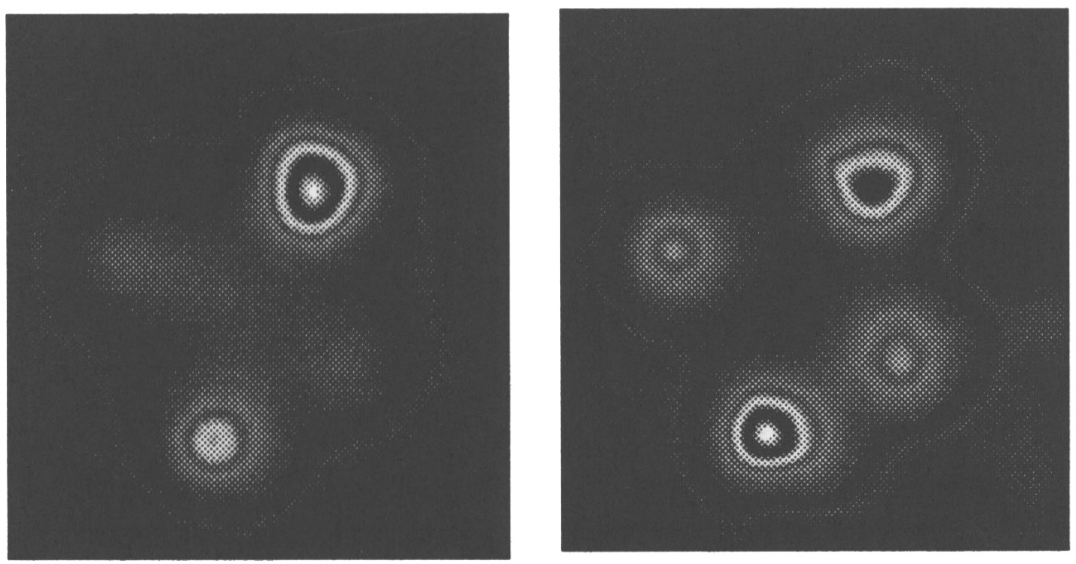

Figure 3. Reconstructed frames of the Einstein cross. Left panel: Total flux in the 5000-7000 $\AA$ wavelength range. Right panel: Continuum free CIII] $1909 \AA$ emission line.

\subsection{The active galaxy NGC 5929}

In this second example we show how to use the 2D spatial sampling to disentangle the spectral properties of the ionized gas in the galaxy. The following results are taken from Pécontal and Ferruit $(1994,1995)$.

A quick look at the object's spectra shows that the emission lines are doublepeaked at some locations (Fig.4). A non-linear Gaussian fit allows a simple decomposition in respectively blue and red shifted components. However at other locations, the double peaked structure is not obvious, and only faint blue or red wings are present. Even at high signal to noise ratio, this non-linear fitting does not guarantee the uniqueness of the solution ${ }^{4}$.

The use of true 2D spatial mapping helps in selecting the physical solution amongst the various numerical ones. For instance a bad fitting solution should be obvious when the image of the line intensity is reconstructed since it will generally not be compatible with the spatial resolution of the observations. In practice the high correlation between neighbouring spectra due to seeing imposed strong constraints on the variation of all physical properties derived from the non-linear fit (line intensities, velocities, line ratios, line widths).

In the case of NGC 5929, Pécontal and Ferruit showed that the blue and red component vanish, but at different locations. The authors were then able to recover the emission line properties (intensity map, map of the various emission line ratio, velocity field, etc...) of each gas cloud. Fig. 5 shows that these clouds are clearly associated with the radio lobes. However, for more complex structures like those present in e.g. NGC 1068, these decomposition techniques require high

\footnotetext{
${ }^{1}$ The best $\chi^{2}$ numerical solution may not be the right one because of noise and/or inaccurate line model (e.g. Gaussian shape)
} 


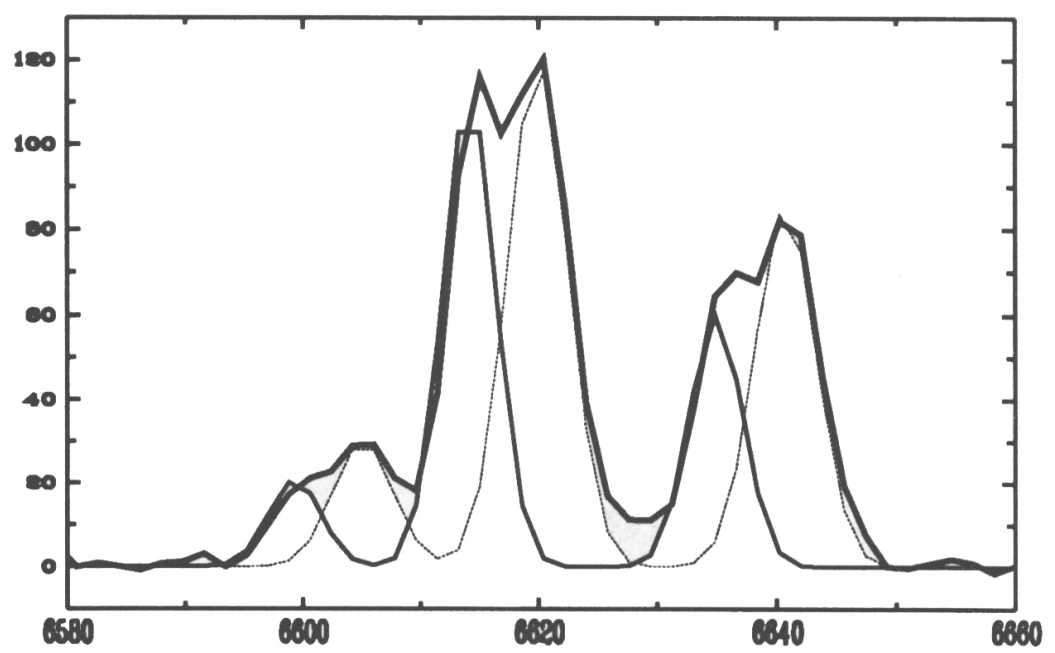

Figure 4. Spectrum of NGC 5929 displaying the double peaked structure and its corresponding Gaussian decomposition.
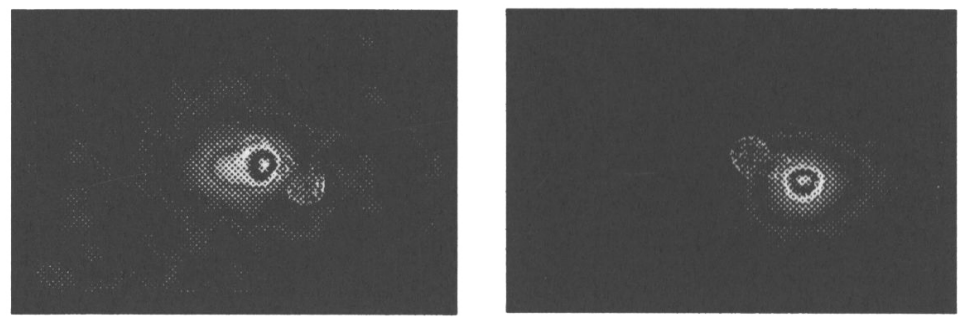

Figure 5. Reconstructed frame of the East and West gaseous emission of NGC 5929. Isointensities of radio $6 \mathrm{~cm}$ map are superimposed. Left panel: East blue shifted component. Right panel: West right shifted component.

spatial resolution to be efficient. The spatial resolution given by TIGER $(\sim 0.7$ arcsec.) and later by OASIS ( $\sim 0.1-0.3$ arcsec. $)$ is crucial for such observations.

\section{Prospects}

Presently, the Observatoire de Lyon, with the Observatoire de Marseille and CFHT, is building a multi-mode integral field spectrograph dedicated to the CFHT adaptive optics bonnette under construction. Named OASIS for Optically Adaptive System for Imaging Spectrography, it features 6 observing modes:

- The Imaging mode, with a maximum 90 arcsec. diameter field and with scales down to 0.025 arcsec. per pixel. 


\begin{tabular}{|c|c|c|c|c|c|c|}
\hline Program & Scientific goals & $\begin{array}{l}\text { OASIS } \\
\text { modes }\end{array}$ & $\begin{array}{l}\text { Typical } \\
\text { size } \\
\text { (arcsec) }\end{array}$ & $\begin{array}{l}\text { Spatial } \\
\text { sampling } \\
\text { (arcsec) } \\
\end{array}$ & $\begin{array}{l}\text { Spectral } \\
\text { range } \\
(\mu \mathrm{m})\end{array}$ & $\begin{array}{l}\text { Spectral } \\
\text { resolution }\end{array}$ \\
\hline Mars & $\begin{array}{l}\text { Mineral composition, } \\
\text { spatial variations }\end{array}$ & $\begin{array}{l}\text { TIGER } \\
\text { ARGUS }\end{array}$ & 15 & $0.1-0.5$ & $0.7-1$ & $500-1500$ \\
\hline Io & $\begin{array}{l}\text { Mineralogy, geology } \\
\text { opatial and temporal } \\
\text { variations }\end{array}$ & $\begin{array}{l}\text { TIGER } \\
\text { ARGUS }\end{array}$ & 1 & 0.04 & $0.5-1$ & $500-3000$ \\
\hline Titan & $\begin{array}{l}\text { Troposphere } \\
\text { structure }\end{array}$ & $\begin{array}{l}\text { TIGER } \\
\text { ARGUS } \\
\end{array}$ & 0.8 & 0.04 & $0.5-1$ & $500-3000$ \\
\hline Comets & $\begin{array}{l}\text { Nucleus structure, } \\
\text { gas, dust, } \\
\text { anisotropy }\end{array}$ & $\begin{array}{l}\text { TIGER } \\
\text { ARGUS }\end{array}$ & $\begin{array}{l}10- \\
\text { few } \\
\text { arcmin }\end{array}$ & $0.1-0.5$ & $0.5-1$ & $500-3000$ \\
\hline $\begin{array}{l}\text { Herbig- } \\
\text { Haro } \\
\text { objects }\end{array}$ & $\begin{array}{l}\text { Density distribution, } \\
\text { dynamics, shocks }\end{array}$ & $\begin{array}{l}\text { TIGER } \\
\text { PYTHEAS } \\
\text { ARGUS } \\
\text { SFP }\end{array}$ & 25 & $0.1-0.5$ & $\begin{array}{l}{[\mathrm{OIII}]} \\
{[\mathrm{NII}]} \\
\mathrm{H} \alpha \\
{[\mathrm{SII}]}\end{array}$ & $\begin{array}{l}3000- \\
10000\end{array}$ \\
\hline $\begin{array}{l}\text { Planetary } \\
\text { nebulae }\end{array}$ & $\begin{array}{l}\text { Emission structure, } \\
\text { dynamics }\end{array}$ & $\begin{array}{l}\text { TIGER } \\
\text { PYTHEAS } \\
\text { ARGUS } \\
\text { SFP }\end{array}$ & $\begin{array}{l}15- \\
\text { few } \\
\text { arcmin }\end{array}$ & $0.1-0.5$ & $\begin{array}{l}{[\mathrm{OIII}]} \\
{[\mathrm{NII}]} \\
\mathrm{H} \alpha\end{array}$ & $\begin{array}{l}3000- \\
20000\end{array}$ \\
\hline $\begin{array}{l}\text { Globular } \\
\text { clusters }\end{array}$ & $\begin{array}{l}X \text { signature } \\
\text { H } \alpha \text { cusp emission }\end{array}$ & $\begin{array}{l}\text { PYTHEAS } \\
\text { (TIGER) } \\
\text { (ARGUS) }\end{array}$ & 15 & $0.1-0.5$ & $0.5-0.7$ & 15000 \\
\hline $\begin{array}{l}\text { Active } \\
\text { nuclei }\end{array}$ & $\begin{array}{l}\text { Ionization, emission } \\
\text { structure, dynamics }\end{array}$ & $\begin{array}{l}\text { TIGER } \\
\text { PYTHEAS } \\
\text { ARGUS } \\
\text { SFP }\end{array}$ & 10 & $0.1-0.5$ & 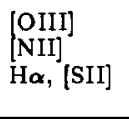 & 3000 \\
\hline $\begin{array}{l}\text { Stellar } \\
\text { dynamics }\end{array}$ & $\begin{array}{l}\text { Dynamics, } \\
\text { 3D stellar } \\
\text { distribution }\end{array}$ & $\begin{array}{l}\text { TIGER } \\
\text { (PYTHEAS) }\end{array}$ & 10 & $0.1-0.5$ & $0.5,0.86$ & 3000 \\
\hline $\begin{array}{l}\text { Interacting } \\
\text { galaxies }\end{array}$ & $\begin{array}{l}\text { Emission, } \\
\text { shocks }\end{array}$ & $\begin{array}{l}\text { TIGER } \\
\text { ARGUS }\end{array}$ & 60 & $0.1-0.5$ & $\begin{array}{l}{[\mathrm{OIII}]} \\
{[\mathrm{NII}]} \\
\mathrm{H} \alpha,[\mathrm{SII}]\end{array}$ & 3000 \\
\hline $\begin{array}{l}\text { Gravita- } \\
\text { tional lenses }\end{array}$ & $\begin{array}{l}\text { Morphology } \\
\mu \text {-amplifification }\end{array}$ & $\begin{array}{l}\text { TIGER } \\
\text { ARGUS }\end{array}$ & $1-5$ & 0.1 & $0.5-1$ & $500-3000$ \\
\hline $\begin{array}{l}\text { Radio- } \\
\text { galaxies }\end{array}$ & $\begin{array}{l}\text { Stars formation } \\
\text { rate, gas structure } \\
\text { and dynamics }\end{array}$ & $\begin{array}{l}\text { TIGER } \\
\text { ARGUS }\end{array}$ & $10-30$ & $0.1-0.5$ & $0.5-1$ & $500-3000$ \\
\hline Quasars & $\begin{array}{l}\text { Morphology and } \\
\text { kinematics of } \\
\text { the extensions }\end{array}$ & $\begin{array}{l}\text { TIGER } \\
\text { ARGUS }\end{array}$ & $10-20$ & $0.1-0.5$ & $0.5-1$ & $500-3000$ \\
\hline
\end{tabular}

Table 3. Examples of OASIS scientific programs. 
- The TIGER mode, with 1400 lenses, $\sim 1.3$ to 16 arcsec. field, down to 0.05 arcsec. sampling and $R$ (spectral resolution) $\leq 3000$.

- The ARGUS mode 5 , with 635 fibers, $\sim 2$ to 5 arcsec. fields, down to 0.1 arcsec. sampling and $R \leq \mathbf{1 5 0 0}$.

- The PYTHEAS scanning mode ${ }^{6}$, with the same fields and scales than TIGER and $R \leq 20,000$.

- The Scanning Fabry-Perot mode ${ }^{7}$, with the same fields and scales than the Imaging mode and $R$ up to 50,000 in a small wavelength range.

- The Long-slit (1D) mode, with a 90 arcsec. slit, down to 0.1 arcsec. sampling and $R \leq 1500$.

OASIS operates in the 0.4-1 $\mu \mathrm{m}$ wavelength range. Different scales are available in each mode through the motion of small optics in the first spatial stage of the instrument. This multi-mode approach has been chosen to make an optimal use of the widely different image qualities that will be delivered by the adaptive optics, depending on the seeing, wavelength, magnitude and location of the reference star. OASIS will be in operation at CFHT at the end of 1996, one year after the Adaptive optics bonnette.

Unlike the case for direct imaging (at least in the visible), OASIS will offer to the CFHT community new capabilities still unmatched by those of the HST. The large scientific domain which could be addressed by OASIS (see some examples in Tab.6.) highlights its complementarity with the HST imaging capabilities.

Acknowledgments. I am grateful to F. Durret, P. Pinet, B. Rocca and other first-time observers, who took the risk of using such a strange machine giving these funny data.

\section{Discussion}

Dr. Ohtani: In the TIGER mode, overlapping of spectra is avoided at the expanse of the blanking area of the detector. This will be a severe problem in cases of IR wavelengths because the costs of IR detector are very high. How do you think to reduce the blank area of the detector?

$R$. Bacon: There is no way to significantly reduce this area (the $\delta$ parameter in Sect.2.). This is an intrinsic limitation of the TIGER design. However this is the price to pay to get a clean separation between spectrographic and spatial information, a property which is essential to get high spatial resolution data.

Dr. Jockers: What is the advantage of the microlenses as compared to an ordinary slit mask?

\footnotetext{
${ }^{5}$ See the review of $\mathrm{C}$. Vanderriest in this proceedings

${ }^{6}$ See the review of $G$. Comte in this proceedings

${ }^{7}$ See the review of P.D. Atherton in this proceedings
} 
R. Bacon: Multi-slits spectrograph are devoted to spectrographic studies on a diluted collection of objects like a cluster of stars or galaxies (that is, discontinuous and randomly located objects). IFS spectrographs like TIGER are devoted to study an extended object (or, nearly equivalent, a dense stellar system). There are very few application overlaps between these two type of instruments.

Dr. Seifert: How do you perform the substraction of the sky background?

$R$. Bacon: When the object does not cover the whole field of view, the average sky spectrum is extracted from the empty regions (manually or using automatic algorithms). When the object is larger than the field, an exposure before and after the object is necessary. However $O H$ emission lines can change very rapidely and this method is not very accurate. By the way, if the object is redshifted, it is possible to take into account the night sky emission lines using an adapted fitting scheme.

Dr. Maillard: Why do you keep a long slit mode in OASIS knowing the limitations of this mode?

$R$. Bacon: This was a preliminary requirement of the CFHT Scientific Advisory Council. It may be useful in some peculiar case when the object geometry is well known and simple enough (e.g. a double star?).

\section{References}

Bacon, R., Adam, G., Baranne, A., Courtès, G., Dubet, D., Dubois, J.P., Georgelin, Y., Monnet, G., Pécontal, E., Urios, J. in ESO Conf. on VLTs and their instrumentation, Ulrich M.H.: ESO, 1989, vol.2, 1185

Bacon, R., Adam, G., Baranne, A., Courtès, G., Dubet, D., Dubois, J.P., Emsellem, E., Ferruit, P., Georgelin, Y., Monnet, G., Pécontal, E., Rousset, A., Sayède, F. 1995, A\&A, in preparation

Courtès, G. in Instrumentation for Astronomy with Large Optical Telescopes, Humphries C.M.: Reidel, 1982, 123

Courtès, G., Georgelin Y., Bacon R., Monnet G., Boulesteix J. in Instrumentation for ground based optical astronomy, Robinson: Springer-Verlag, 1988, 267

Fitte, C., Adam, G. 1994, A\&A, 282, 11

Pécontal, E., Ferruit, P. in Multi-Wavelength continuum emission of AGN, IAU symp. 159, Courvoisier T.J. and Blecha A.: Kluwer acad. pub., 1994, p445.

Pécontal, E., Ferruit, P., 1995, A\&A, in preparation

Rousset, A., Bacon, R., Pécontal, E., Emsellem, E., Ferruit, P., Adam, G., 1995, A\& $\mathrm{A}$, in preparation 